\title{
JIP1 binding to RBP-Jk mediates cross-talk between the Notch1 and JIP1-JNK signaling pathway
}

\author{
M-Y Kim ${ }^{1,5}$, E-J Ann ${ }^{1,5}$, J-S Mo ${ }^{1,5}$, F Dajas-Bailador ${ }^{2}$, M-S Seo ${ }^{1}$, J-A Hong', J Jung', Y-H Choi', J-H Yoon ${ }^{1}$, S-M Kim', E-J Choi, \\ H-S Hoe ${ }^{4}$, AJ Whitmarsh ${ }^{2}$ and H-S Park ${ }^{* 1}$
}

Notch1 signaling has a critical function in maintaining a balance among cell proliferation, differentiation, and apoptosis. Our earlier work showed that the Notch1 intracellular domain interferes with the scaffolding function of $c$-Jun $\mathrm{N}$-terminal kinase (JNK)-interacting protein-1 (JIP1), yet the effect of JIP1 for Notch1-recombining binding protein suppressor of hairless (RBP-Jk) signaling remains unknown. Here, we show that JIP1 suppresses Notch1 activity. JIP1 was found to physically associate with either intracellular domain of Notch1 or RBP-Jk and interfere with the interaction between them. Furthermore, we ascertained that JIP1 caused the cytoplasmic retention of RBP-Jk through an interaction between the C-terminal region of JIP1 including SrC homology 3 domain and the proline-rich domain of RBP-Jk. We also found that RBP-Jk inhibits JIP1-mediated activation of the JNK1 signaling cascade and cell death. Our results suggest that direct protein-protein interactions coordinate cross-talk between the Notch1-RBP-Jk and JIP1-JNK pathways.

Cell Death and Differentiation (2010) 17, 1728-1738; doi:10.1038/cdd.2010.50; published online 28 May 2010

Signaling by Notch is associated with a variety of cellular events, including cell-fate control and pattern formation during embryonic development, cell differentiation, cell survival, and cell death in vertebrates and invertebrates. ${ }^{1,2}$ Mammals express four Notch genes (Notch1-Notch4) and five ligands for Notch from two conserved families, Jagged (JAG1 and JAG2) and Delta (DLL1, DLL3, and DLL4) in different combinations in most cell types. After ligand binding, Notch proteins are activated by a series of cleavages that release its intracellular domain (Notch-IC), followed by its nuclear translocation. ${ }^{3}$ The nuclear translocation of Notch-IC results in the transcriptional activation of genes of the HESs family [Hes/E(spl) family] and HEYs family (Hesr/Hey family) through the interaction of Notch-IC with CSL (CBF1/recombining binding protein suppressor of hairless (RBP-Jk) in vertebrates, Suppressor of Hairless [SuH] in Drosophila melanogaster, Lag-1 in Caenorhabditis elegans, and through mastermind-like (MAML)). ${ }^{2,3}$ In the absence of the Notch-IC, RBP-Jk acts as a transcriptional repressor because of its ability to bind transcriptional corepressors and histone deacetylase-1. Notch-IC translocates to the nucleus in which it interacts with the RBP-Jk and displaces corepressor complexes and recruits coactivators, thus turning RBP-Jk into a transcriptional activator. ${ }^{4}$

c-Jun N-terminal kinase (JNK)-interacting protein-1 (JIP1) was first identified as a scaffold for JNK signaling from a yeast two-hybrid screen, facilitating mixed-lineage kinase
(MLK)-dependent JNK signal transduction by aggregating the three components of the module, MLK, MKK7, and JNK, on different regions of JIP1.,6 JIP proteins can form homo- and hetero-oligomeric complexes with components of the JNK signaling pathway. In addition, JIP1 interacts with Alzheimer's amyloid precursor protein, ${ }^{7}$ p190rhoGEF, ${ }^{8}$ light chain of kinesin, ${ }^{9}$ apolipoprotein $\mathrm{E}$ receptor $2,{ }^{10}$ T-lymphokine-activated killer cell-originated protein kinase ${ }^{11}$ the vaccinia virus $\mathrm{B} 1 \mathrm{R}$ kinase, ${ }^{12}$ vaccinia-related kinase- $2,{ }^{13}$ protein kinase B/Akt, ${ }^{14}$ Src family kinase, ${ }^{15}$ TGF- $\beta$-activated kinase- $1,{ }^{16}$ and the intracellular domain of Notch1 (Notch1-IC). ${ }^{17}$ The interaction of JIP1 with a variety of proteins suggests that JIP1 serves as a multifunctional protein.

We earlier showed that Notch1-IC, an active form of Notch1 generated by the action of $\gamma$-secretase, functions as a negative regulator of JNK signaling by disrupting the scaffold function of JIP1. ${ }^{17}$ Despite these observations, the precise mechanisms underlying the connection between Notch and JIP1-JNK signaling pathways remain to be accurately delineated. Therefore, in this study, we evaluated the molecular basis of the signal cross-talk occurring between them. JIP1 suppresses Notch1-IC-mediated signaling through disruption of the Notch1-RBP-Jk complex. Interestingly, the down-regulation of Notch1 signaling was found to be the consequence of the cytoplasmic retention of RBP-Jk through physical interaction with JIP1. Furthermore, we found that the ectopic expression of RBP-Jk inhibited JIP1-mediated

\footnotetext{
${ }^{1}$ Hormone Research Center, School of Biological Sciences and Technology, Chonnam National University, Gwangju, Republic of Korea; ${ }^{2}$ Faculty of Life Sciences, University of Manchester, Manchester, UK; ${ }^{3}$ School of Life Sciences and Biotechnology, Korea University, Seoul, Republic of Korea and ${ }^{4}$ Department of Neuroscience, Georgetown University Medical Center, Washington, DC, USA

*Corresponding author: H-S Park, School of Biological Sciences and Technology, Chonnam National University, 300 Yongbong-dong, Bukku, Gwangju 500-757, Republic of Korea. Tel: + 8262530 0021; Fax: + 8262530 2199; E-mail: proteome@jnu.ac.kr

${ }^{5}$ These authors contributed equally to this work.

Keywords: RBP-Jk; Notch1; JIP1; SH3 domain; cytoplasmic retention

Abbreviations: RBP-Jk, recombining binding protein suppressor of hairless; JIP1, JNK-interacting protein-1; JNK, c-Jun N-terminal kinase; SH3 domain, Src homology 3 domain

Received 02.11.09; revised 15.3.10; accepted 14.4.10; Edited by H Ichijo; published online 28.5.10
} 
activation of JNK1 signaling and cell death induced by glucose deprivation. Collectively, our findings show that there is feedback regulation between the Notch1-RBP-Jk and JIP1JNK pathways through protein-protein interaction and thereby regulate cell-fate decisions, differentiation, proliferation, and cell death and survival.

\section{Results}

JIP1 suppresses the Notch1 signaling pathway. We have earlier shown that Notch1-IC suppresses the JIP1mediated JNK signaling cascade and cell death through physical association. ${ }^{17}$ Inversely, to determine whether JIP1 is involved in the regulation of Notch1 signaling, a transcriptional reporter assay was performed using Notch1-dependent promoter elements linked to the luciferase gene. The Notch1-IC-induced luciferase reporter activity was inhibited by the cotransfection of JIP1 in HEK293 (Figure 1a) and NIH3T3 cells (Figure 1b). To determine the function of endogenous JIP1 in Notch1 signaling, we performed a transcription reporter assay using JIP1 wild-type $\left(\mathrm{JIP}^{+/+}\right)$and JIP1-deficient $\left(\mathrm{JIP1}^{-/-}\right)$fibroblast cells. The transcriptional activity of Notch1-IC in JIP1deficient fibroblasts was threefold higher than in JIP1 wild-type cells (Figure 1c). We also found that Notch1-IC transcriptional activity was suppressed by overexpressed JIP1 in JIP1-deficient cells (Figure 1d). These results indicated that JIP1 suppresses the transcriptional activity of Notch1 in intact cells.

JIP1 prevents physical association between Notch1-IC and RBP-Jk. Our further findings indicated that the JIP1induced suppression of Notch1 activity was not significantly correlated with the inhibition of the $\gamma$-secretase-dependent proteolytic cleavage of the active Notch1 mutant $\Delta \mathrm{EN} 1$ to Notch1-IC (Figure 2a). In addition, the steady state protein level of $\Delta \mathrm{EN} 1$ and Notch1-IC were not diminished in these cases (Figure 2a), suggesting that, at least in part, the JIP1-induced inhibition of Notch1 can be linked neither to the regulation of cleavage patterns, nor to protein stability. To determine the effects of JIP1 on the molecular interactions occurring between Notch1-IC and RBP-Jk, coimmunoprecipitation was conducted in HEK293 cells after the cotransfection of vector encoding Myc-tagged Notch1-IC, Flag-tagged RBP-Jk, and HA-tagged JIP1. The results indicated that JIP1 interrupts the physical association between Notch1-IC and RBP-Jk (Figure 2b and $c$ ). To further substantiate this finding, coimmunoprecipitations were performed in JIP1 wild-type and JIP1-deficient fibroblast cells. Endogenous binding between Notch1-IC and RBP-Jk in JIP1-deficient fibroblasts was much higher than in JIP1 wild-type cells (Figure 2d). These results suggest that JIP1 may negatively regulate the transactivation of Notch1-IC target genes, through the suppression of the interaction between Notch1-IC and RBP-Jk in intact cells. a

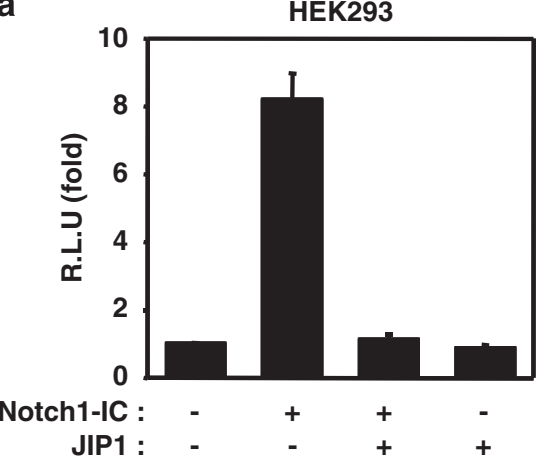

C

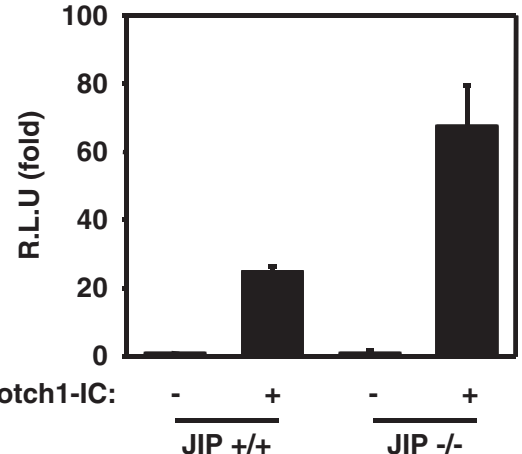

b

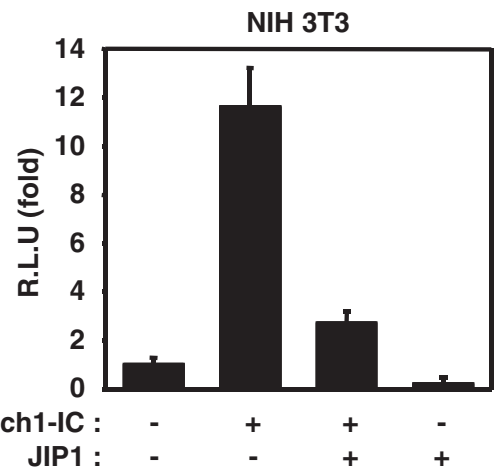

d

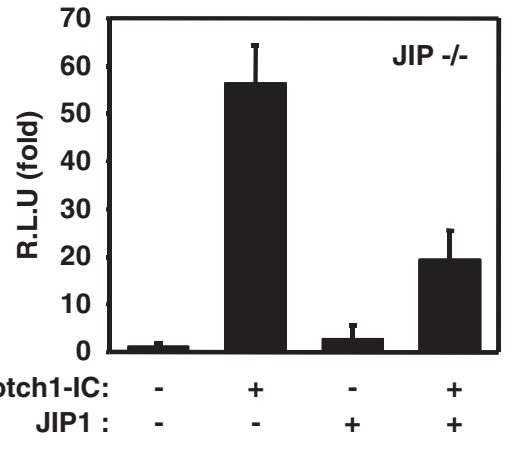

Figure 1 JIP1 suppresses the Notch1 signaling pathway. (a) HEK293 or (b) NIH3T3 cells were transfected with expression vectors for 4XCSL-Luc, and $\beta$-galactosidase, along with Notch1-IC and JIP1, as indicated. (c) JIP1 ${ }^{+1+}$ and JIP1 $1^{-1-}$ MEF cells were transfected with expression vectors for 4XCSL-Luc, and $\beta$-galactosidase, along with Notch1-IC. (d) JIP1 ${ }^{-l-}$ cells were transfected with expression vectors for 4XCSL-Luc, and $\beta$-galactosidase, along with Notch1-IC and JIP1. After $48 \mathrm{~h}$ of transfection, the cells were lysed, and the luciferase activity was determined. The data was normalized with $\beta$-galactosidase. These results represent the means \pm average deviation of three independent experiments. RLU means relative luciferase units 
a

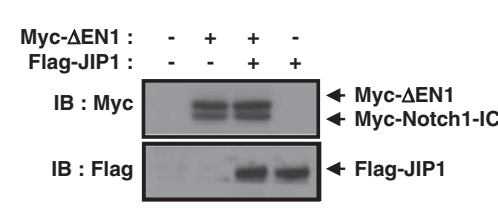

Myc-Notch1-IC : $\quad-\quad+\quad+$

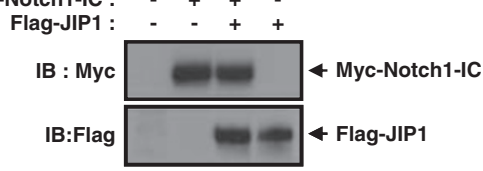

C

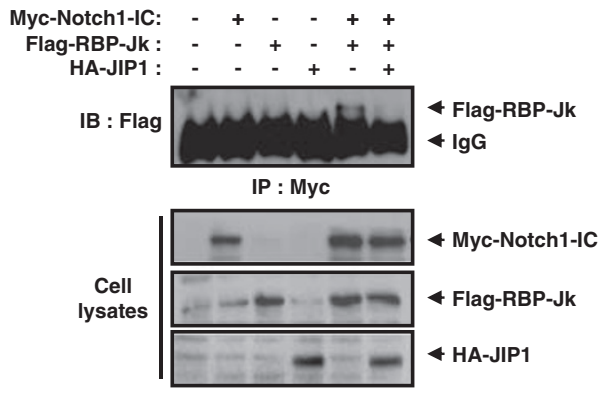

b

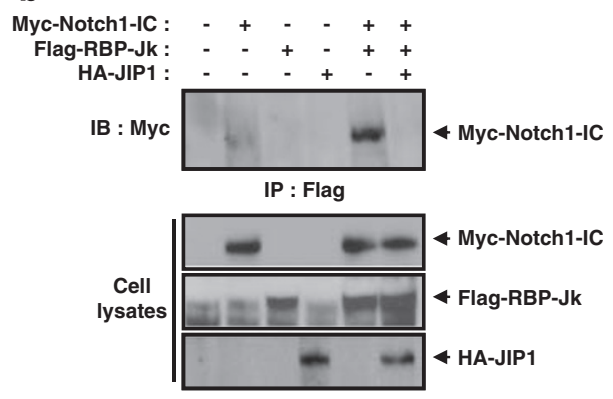

d
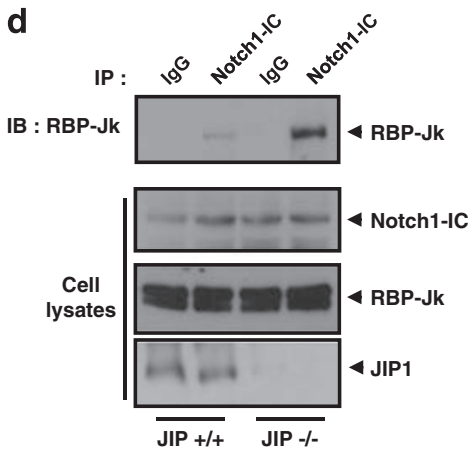

Figure 2 JIP1 prevents physical association between Notch1-IC and RBP-Jk. (a) HEK293 cells were transfected with expression vectors for Myc- $\Delta$ EN1 and Flag-JIP1. HEK293 cells were transfected with expression vectors for Myc-Notch1-IC and JIP1-Flag. The cell lysates were subjected to immunoblot analysis with anti-Myc and anti-Flag antibodies, respectively. (b, c) HEK293 cells were transfected with expression vectors for Myc-Notch1-IC, Flag-RBP-Jk, and HA-JIP1, as indicated. (b) Cell lysates were subjected to immunoprecipitation with anti-Flag antibody and the immunoprecipitates were immunoblotted with anti-Myc antibody. (c) Cell lysates were subjected to immunoprecipitation with anti-Myc antibody and the immunoprecipitates were immunoblotted with anti-Flag antibody. The cell lysates were also subjected to immunoblot analysis with anti-Myc, anti-Flag, and anti-HA antibodies, respectively. (d) JIP1 ${ }^{+1+}$ and JIP1 $1^{-1-}$ MEF cells were lysed and subjected to immunoprecipitation with IgG and anti-Notch1-IC antibodies as indicated. Immunoprecipitates were immunoblotted with anti-RBP-Jk antibody. The cell lysates were immunoblotted with anti-Notch1-IC, anti-RBP-Jk, and anti-JIP1 as controls

JIP1 sequesters RBP-JK in the cytoplasm. Notch1-IC and RBP-Jk are localized mainly in the nucleus, and as shown in Figure $3 a$, the intracellular distribution of JIP1 is in the cytoplasm. To determine the effect of JIP1 on Notch1-IC and RBP-Jk localization, cells were cotransfected with vector encoding HA-JIP1, Flag-RBP-Jk, and Myc-Notch1-IC. The expression of JIP1 significantly blocked the translocation of RBP-Jk into the nucleus and about $90 \%$ of RBP-Jk remained in the cytoplasm (Figure 3a). However, JIP1 did not affect the cellular localization of Notch1-IC, which was distributed in the nucleus. We found similar results using JIP1 knockout cell lines. The overexpression of JIP1 in the $\mathrm{JIP} 1^{-1-}$ cells prohibited nuclear accumulation of RBP-Jk (Figure 3b). The results of this experiment suggest that the inhibitory function of JIP1 in the Notch1 signaling pathway is mediated by regulating RBP-Jk subcellular localization.

\section{JIP1 interacts with RBP-Jk and Notch1-IC and forms a} trimetric complex. Earlier we have shown that JIP1 may interact with Notch $1-\mathrm{IC},{ }^{17}$ and Notch1-IC was mainly present in the nucleus and moderately present in the cytoplasm. ${ }^{17-19}$ To delineate more precisely the manner in which JIP1 prevents Notch1-IC and RBP-Jk-mediated transcription, we conducted a series of in vitro binding and coimmunoprecipitation experiments. In the in vitro binding studies, an interaction between GST-RBP-Jk and JIP1 was detected on the bead complexes (Figure 4a). To further characterize the physical interaction occurring between JIP1 and RBP-Jk, coimmunoprecipitation was conducted in the HEK293 cells through the cotransfection of vector encoding Flag-RBP-Jk or HA-JIP1. The results indicated that JIP1 interacts with RBP-Jk (Figure 4b). In addition, using cytoplasmic fraction of mouse brain extract, PC12 cells and SH-SY5Y cells, we found endogenous binding not only between Notch1-IC and JIP1, but also between RBP-Jk and JIP1 (Figure 4c). Furthermore, we also confirm the endogenous binding between these proteins using $\mathrm{JIP} 1^{+/+}, \mathrm{JIP} 1^{-/-}$, and JIP1 introduced JIP1 ${ }^{-1-}$ cells (Figure 4d). From these results, we could postulate that JIP1 forms a trimeric complex with Notch1-IC and RBP-Jk. To confirm this, HEK293 cells were cotransfected with vectors encoding Myc-Notch1-IC, Flag-RBP-Jk, and HA-JIP1, and were then subjected to coimmunoprecipitation analysis using cytoplasmic fraction of cell lysates. Immunoblot analysis revealed that JIP1 formed a trimeric complex with Notch1-IC and RBP-Jk (Figure 4e). Next, we examined whether endogenous JIP1 could form a trimeric complex in cytoplasmic fraction of mouse brain extracts. Immunoblot analysis indicated the trimeric formation of endogenous JIP1, Notch1-IC, and RBP-Jk (Figure 4f). In the trimeric complex, we questioned whether JIP1 could bind RBP-Jk without Notch1-IC, or whether JIP1 could bind Notch1-IC without RBP-Jk. To examine this 


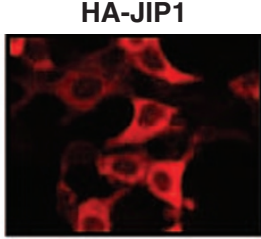

Flag-RBP-Jk

Myc-Notch1-IC

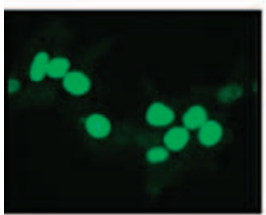

Myc-Notch1-IC

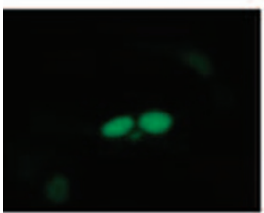

Flag-RBP-Jk

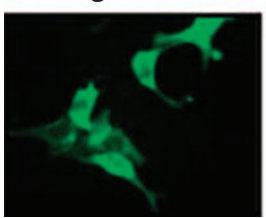

b

JIP-/-

+ HA-RBP-Jk

JIP-/-

+ Flag-JIP1
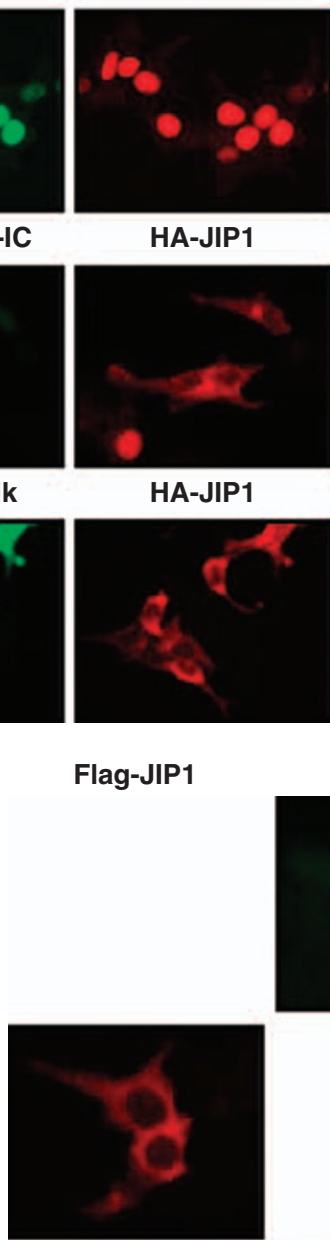

HA-JIP1

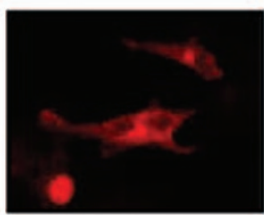

HA-JIP1
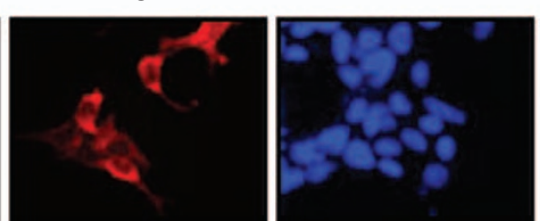

Flag-JIP1

\begin{abstract}
HA-RBP-Jk
\end{abstract}
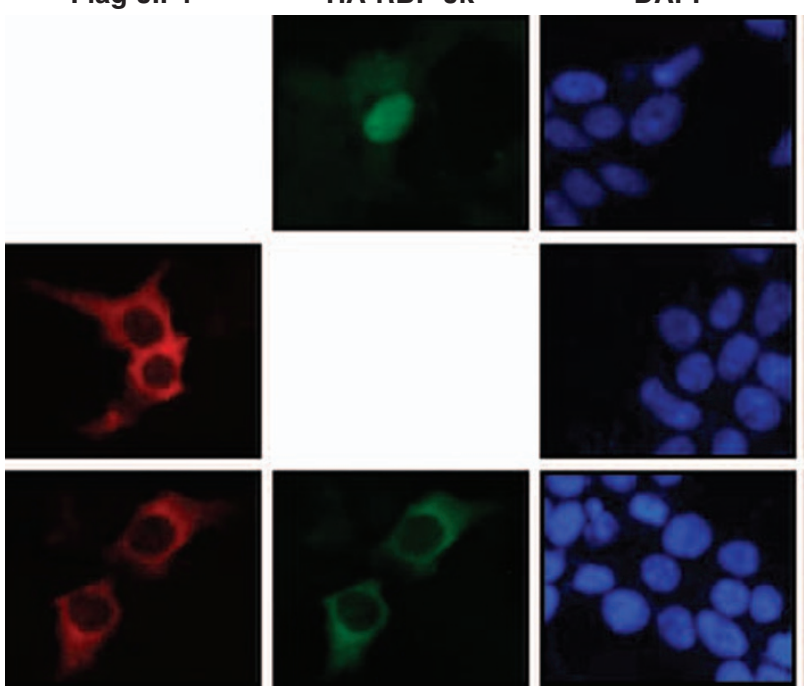

Merge

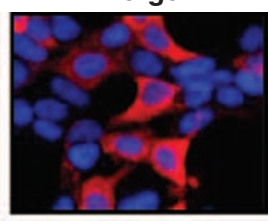

Merge

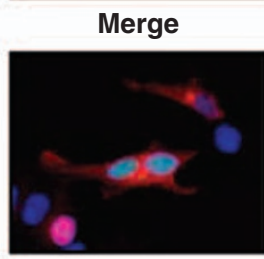

JIP-/-

+ Flag-JIP1

+ HA-RBP-Jk

HA-JIP1

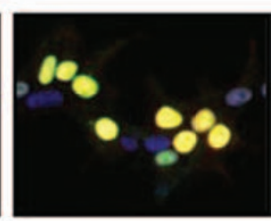

Myc-Notch1-IC + Flag-RBP-Jk

Myc-Notch1-IC + Flag-RBP-Jk + HA-JIP1

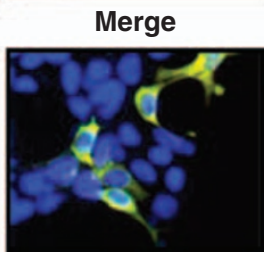

Myc-Notch1-IC + Flag-RBP-Jk + HA-JIP1

Figure 3 JIP1 sequesters RBP-Jk in the cytoplasm. (a) HEK293 cells were transfected with expression vectors for Myc-Notch1-IC, Flag-RBP-Jk, and HA-JIP1, as indicated. The cells were double stained for Notch1-IC (green), RBP-Jk (red or green), and JIP1 (red). (b) JIP1 ${ }^{-l-}$ MEF cells were transfected with expression vector for RBP-Jk and JIP1-Flag, as indicated. Transfected JIP1 ${ }^{-1-}$ MEF cells were double stained for JIP1 (red) and RBP-Jk (green) using anti-Flag and anti-HA antibodies, respectively. (a, b) Nuclei were stained with DAPI. The cells were visualized by confocal microscopy (Leica SPE)

question, we used Notch1-IC- $\triangle \mathrm{RAM}$, which is unable to interact with RBP-Jk. JIP1 still formed complexes with Notch1-IC- $\triangle$ RAM and RBP-Jk, suggesting that JIP1 can bind separately to Notch1-IC and RBP-Jk through perhaps different domains (Figure 4g). Taken together, JIP1 could bind both Notch1-IC and RBP-Jk in the cytoplasm without competition and finally RBP-Jk accumulated in the cytoplasm, but Notch1-IC move into the nucleus under unknown mechanism.

C-terminal region of JIP1 including the Src homology 3 domain is critical for binding to the proline-rich region of RBP-Jk. To address which domain of JIP1 might have an important function in the binding and suppression of Notch1 
signaling, we performed an in vitro binding and coimmunoprecipitation assay using several deletion mutants (Figure 5c). In our earlier report, it was shown that Notch1IC could bind to the JNK-binding domain (JBD) domain of
JIP1 and thereby suppress JIP1-mediated JNK1 signaling pathway. ${ }^{17}$ Here, we investigated which of the domains might be involved in the interaction of JIP1 and RBP-Jk. In vitro binding analysis with GST-fused RBP-Jk revealed

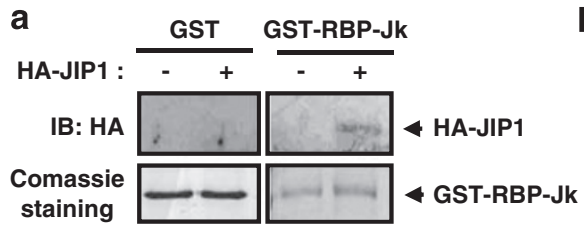

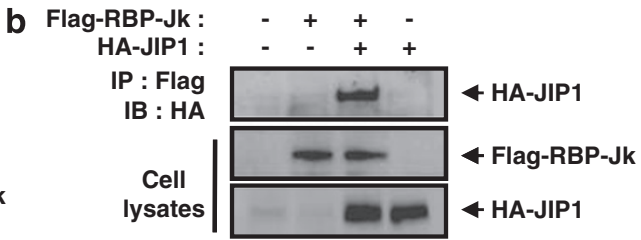

C

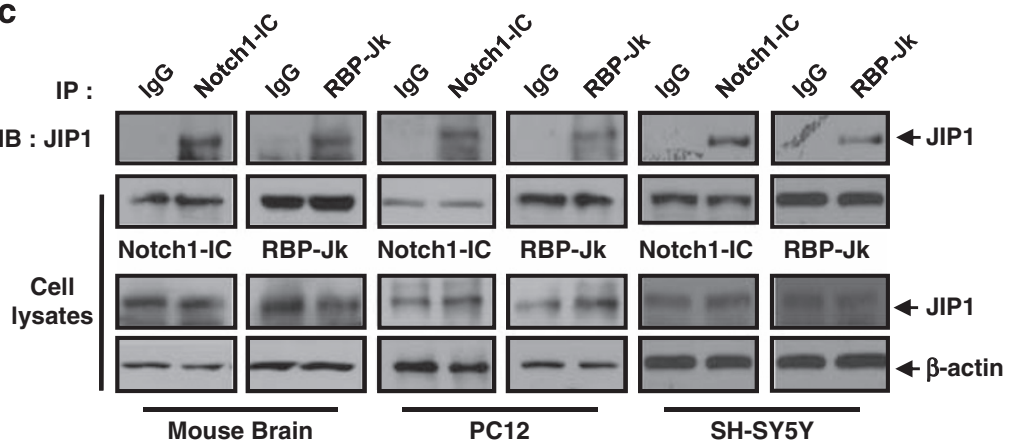

f

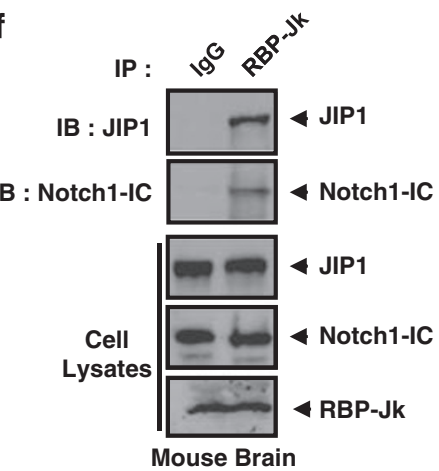

d

d

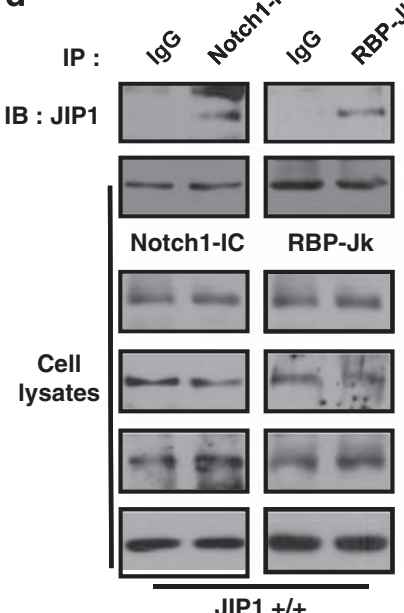

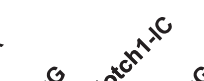

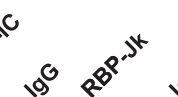
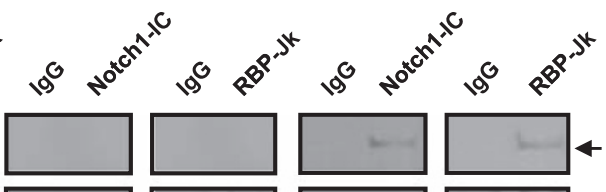

IB : JIP
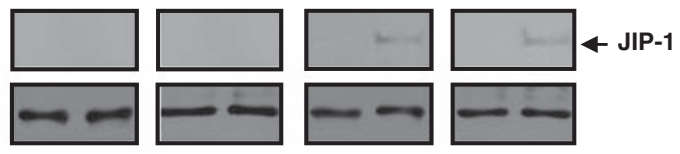

Notch1-IC RBP-Jk
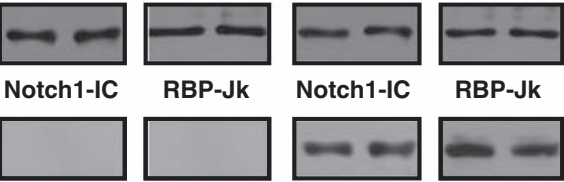

Notch1-IC

RBP-Jk

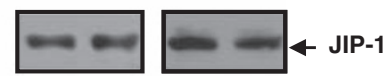

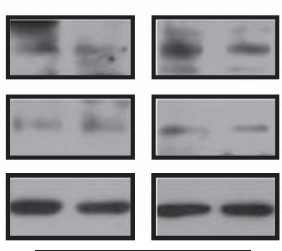

JIP1 -/-

9

Flag-Notch1-IC- $\triangle$ RAM: $\quad-\quad+\quad-\quad+$

Myc-RBP-Jk : $\quad-\quad+\quad+$

HA-JIP1 : $\quad-\quad+++$

$\begin{array}{rllll}\text { Myc-Notch1-IC: } & - & + & - & + \\ \text { Flag-RBP-Jk: } & - & - & + & +\end{array}$

HA-JIP1 : -+++

IB : Myc

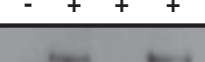

IB : Flag

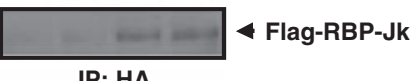

IP: HA

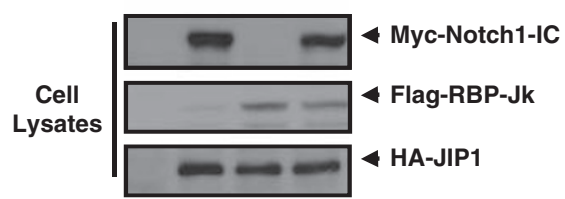

IB : Flag

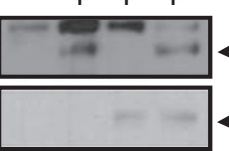

Flag-Notch1-IC- $\triangle$ RAM

IB : Myc

IP: HA

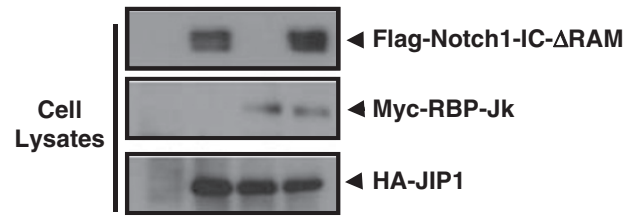


that amino-acids 292-518, including the Src homology 3 (SH3) domain of JIP1, was required for binding to RBP-Jk (Figure 5a). A subsequent coimmunoprecipitation experiment also showed that amino-acids 292-518 of JIP1, including $\mathrm{SH} 3$ domain, were important for binding to RBP-Jk (Figure 5b). Therefore, we could suggest that Notch1-IC interacts with the JBD domain of JIP1 and RBP-Jk interacts with C-terminal domain of JIP1 including $\mathrm{SH} 3$ domain (Figure $5 \mathrm{c}$ ). We then assessed the inhibition of Notch1 activity by the JIP1 deletion mutants, JIP1- $\Delta J B D$, JIP-JBD, and JIP1-phosphotyrosine interaction domain (PID). We found that JIP1- $\triangle$ JBD were able to suppress Notch1-IC-mediated transactivation of the 4XCSL luciferase reporter system (Figure 5d). The PID domain of JIP1 did not participate in binding or suppression of the Notch1 signaling pathway (Figure $5 d$ ).

Earlier reports have shown that RBP-Jk contains a polyproline region, which can serve as a docking site to $\mathrm{SH} 3$ domains. ${ }^{20}$ To address the possible region of RBP-Jk that binds to JIP1, we performed the site-directed mutation of polyproline to alanine within RBP-Jk (amino-acids 95-97). The physical association occurring between JIP1 and RBP-Jk was diminished by the proline to alanine mutation (Figure $5 \mathrm{e}$ ). Furthermore, JIP1 did not alter the RBP-Jk (PPP/AAA) mutant and Notch1-IC-mediated transactivation (Figure 5f). Next, we examined the effect of JIP1 on the subcellular localization of RBP-Jk and RBP-JK (PPP/AAA) mutant. The expression of JIP1 significantly blocked the translocation of RBP-Jk into the nucleus. However, JIP1 did not affect the cellular localization of RBP-Jk (PPP/AAA) mutant, which was distributed in the nucleus (Figure $5 \mathrm{~g}$ ). The overexpression of JIP1 and JIP1$\triangle \mathrm{JBD}$ prohibited nuclear accumulation of RBP-Jk (Figure $5 \mathrm{~h}$ ). Taken together, these data suggest that the physical interaction between SH3 domain of JIP1 and the proline-rich domain of RBP-Jk sequesters RBP-Jk in the cytoplasm and is an important mechanism by which JIP1 suppresses Notch1-IC activation.

RBP-Jk prevents JIP1-mediated signaling and cell death. JIP1 physically associates with MLK3, MKK7, and JNK, and thus functions as a scaffold protein that facilitates JNK activation by the MLK3-MKK7-JNK signaling module. ${ }^{6,21,22}$ To characterize further the mechanism underlying the action of RBP-Jk on the JIP1 pathway, we examined whether RBP-Jk affects JIP1-mediated activation of JNK. Coexpression of JIP1 increased the kinase activity of ectopic JNK1 in HEK293 cells that had been cotransfected with vectors for MLK3, MKK7, and JNK1 (Figure 6a). This scaffold function of JIP1 was inhibited by RBP-Jk. We then examined whether RBP-Jk could alter the binding of JIP1 to MLK3, MKK7, or JNK1. Coimmunoprecipitation analysis revealed that RBP-Jk markedly inhibited interaction between JIP1, and MLK3 and MKK7, and did not affect the interaction between JIP1 and JNK1 (Figure 6b). We then examined the effect of RBP-Jk on the homo-dimerization of JIP1 in transfected HEK293 cells. Coimmunoprecipitation analysis showed that JIP1 proteins were bound to each other and this interaction was effectively inhibited by RBP-Jk (Figure 6c).

Earlier the deprivation of glucose and oxygen in hippocampal neurons has been shown to induce activation of the JNK pathway and consequent apoptosis dependent on JIP1. ${ }^{22}$ JNK1 enzyme activity, which was stimulated by glucose deprevation, was promoted through the down-regulation of Notch1 signaling using $\gamma$-secretase-specific inhibitor DAPT and down-regulation of RBP-Jk expression in JIP1 JIP1 ${ }^{-1-}$ and $\mathrm{JIP} 1^{+/+}$mouse embryonic fibroblast (MEF) cells (Figure $6 \mathrm{~d}-\mathrm{g})$. Similarly, ectopic expression of JIP1 in the JIP1 ${ }^{-1-}$ MEF cells supported glucose deprivation-induced JNK1 activation, which was inhibited by ectopic expression of RBP-Jk (Figure 6h). RBP-Jk expression also inhibited JNK1 activation in wild-type MEF cells (Figure 6i). In contrast, mutant RBP-Jk did not affect the activation of JNK1 induced by JIP1 in the JIP1-introduced JIP1 ${ }^{-1-}$ MEF (Figure 6h) or the wild-type MEF cells (Figure 6i). Furthermore, RBP-Jk, but not mutant RBP-Jk, inhibited glucose deprivation-induced apoptosis in the JIP1 ${ }^{-/-}$MEF cells, as shown by $4^{\prime}, 6$-diamidino-2phenyl-indole dihydrochloride (DAPI) staining (Figure 6j). Glucose deprivation did not induce apoptosis in the JIP1 $1^{-1-}$ MEF cells. Consistent with an earlier report, we found that glucose deprivation-induced cell death was suppressed by Notch1-IC and Notch1-IC- $\triangle$ RAM (Figure 6j). Taken together, these results suggest that RBP-Jk inhibits the activation of JNK and apoptosis mediated by JIP1.

Figure 4 JIP1 interacts with RBP-Jk and Notch1-IC and forms a trimetric complex. (a) Recombinant GST or GST-RBP-Jk proteins were immobilized onto GSH-agarose. HEK293 cells were transfected for $48 \mathrm{~h}$ with the expression vector encoding HA-JIP1 or empty vector. After transfection, the cell lysates were then subjected to GST-pull-down experiments with immobilized GST or GST-RBP-Jk. JIP1 proteins bound to GST or GST-RBP-Jk was analyzed through immunoblotting with anti-HA antibody. GST or GST-RBP-Jk proteins were visualized through staining with Coomassie brilliant blue. (b) HEK293 cells were transfected for $48 \mathrm{~h}$ with expression vectors encoding Flag-RBP$\mathrm{Jk}$ and HA-JIP1, as indicated. After transfection, the cytoplasmic fractions of cell lysates were subjected to immunoprecipitation with anti-Flag antibody. The immunoprecipitates were then immunoblotted with anti-HA antibody. Cell lysates were also immunoblotted with anti-Flag and anti-HA antibodies. (c) Mouse brain, PC12, and SH-SY5Y cells were lysed and the cytoplasmic fractions were subjected to immunoprecipitation with IgG, anti-Notch1-IC, and anti-RBP-Jk antibodies, as indicated. Immunoprecipitates were immunoblotted with anti-JIP1 antibody. Cell lysates were immunoblotted with anti-Notch1-IC, anti-RBP-Jk, and anti- $\beta$-actin as controls. (d) JIP1 $+/+$ and $\mathrm{JIP} 1^{-1-}$ MEF cells were lysed and subjected to immunoprecipitation with IgG, anti-Notch1-IC, and anti-RBP-Jk antibodies as indicated. JIP1 ${ }^{-1-}$ MEF cells were transfected for $48 \mathrm{~h}$ with expression vectors encoding JIP1. Immunoprecipitates were immunoblotted with anti-RBP-Jk antibody. The cell lysates were immunoblotted with antiNotch1-IC, anti-RBP-Jk, and anti-JIP1-3 as controls. (e) HEK293 cells were transfected for $48 \mathrm{~h}$ with expression vectors encoding Myc-Notch1-IC, Flag-RBP-Jk, and HA-JIP1. After transfection, the cytoplasmic fractions of cell lysates were subjected to immunoprecipitation with anti-HA antibody. The immunoprecipitates were then immunoblotted with anti-Myc or anti-Flag antibody. Cell lysates were also immunoblotted with anti-Myc, anti-Flag, and anti-HA antibodies. (f) Mouse brains were lysed and the cytoplasmic fractions were subjected to immunoprecipitation with IgG and anti-RBP-Jk antibodies, as indicated. Immunoprecipitates were immunoblotted with anti-JIP1 and anti-Notch1-IC antibodies. Cell lysates were immunoblotted with anti-JIP1, anti-Notch1-IC, and anti-RBP-Jk. (g) HEK293 cells were transfected for $48 \mathrm{~h}$ with expression vectors encoding Flag-Notch1-IC- $\triangle$ RAM, Myc-RBP-Jk, and HA-JIP1. After transfection, the cytoplasmic fractions of cell lysates were subjected to immunoprecipitation with anti-HA antibody. The immunoprecipitates were then immunoblotted with anti-Flag or anti-Myc antibody. Cell lysates were also immunoblotted with anti-Flag, anti-Myc, and anti-HA antibodies 


\section{Discussion}

In our earlier report, it was shown that Notch1 negatively regulates the JNK signaling pathway and JNK-dependent cell death through physical binding with JIP1. ${ }^{17}$ The Notch1-ICmediated inhibition of JNK signaling seems to require physical interaction, given that Notch1 containing the extracellular cysteine-rich LIN-12/Notch/GLP repeats (Notch1-LNG), which are not converted to Notch1-IC by $\gamma$-secretase, did not inhibit JNK activation. ${ }^{17}$ In contrast, we have now shown that JIP1 can negatively regulate Notch1 signaling by facilitating the accumulation of RBP-Jk in the cytoplasm.

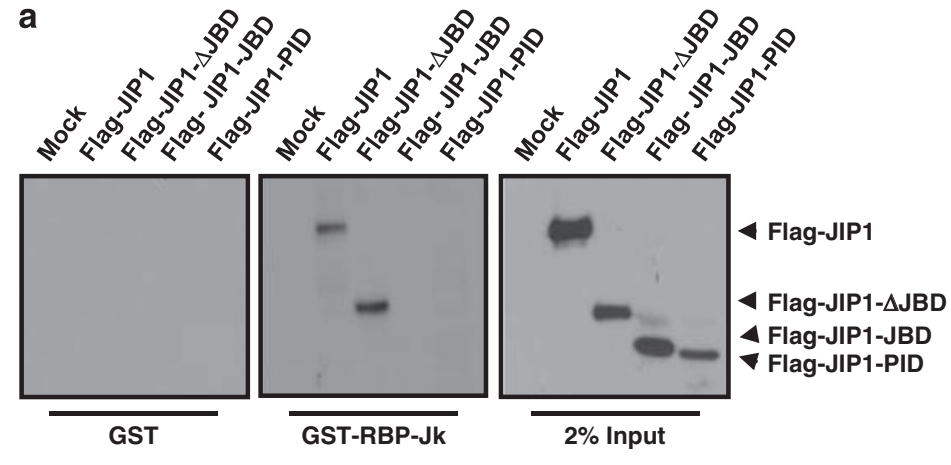

b

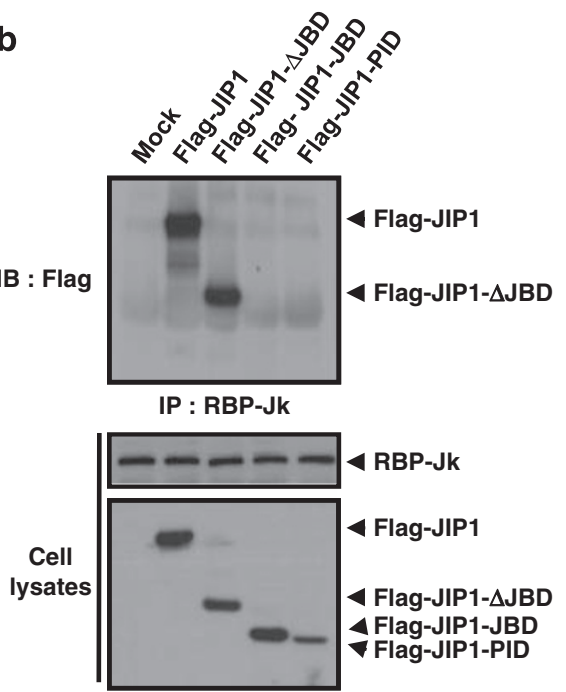

e Flag-RBP-Jk: W W $\quad$ M $\quad M$ HA-JIP1b :

IB : anti-Flag

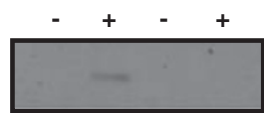

4 Flag-RBP-Jk

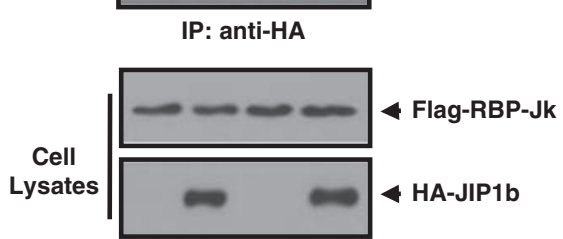

c

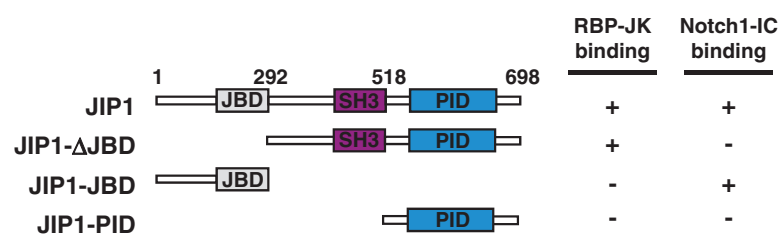

d
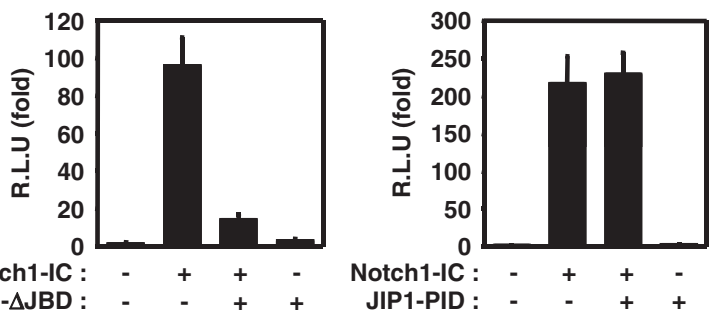

g

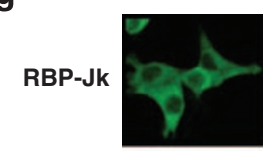

mtRBP-Jk
JIP1
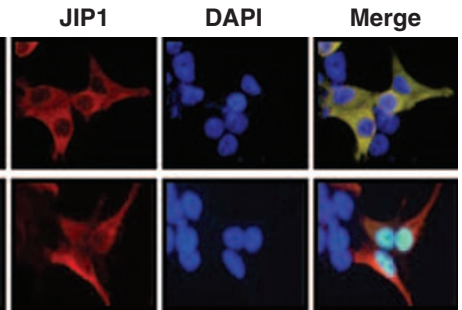

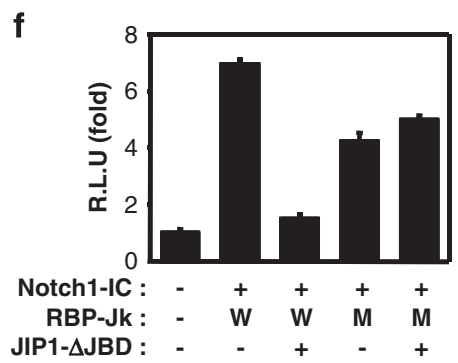

JIP1- $\triangle$ JBD : - $\quad-\quad+\quad+$

h
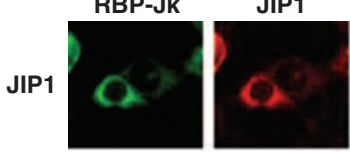

JIP1
DAPI

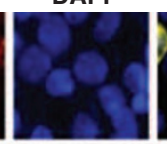

Merge
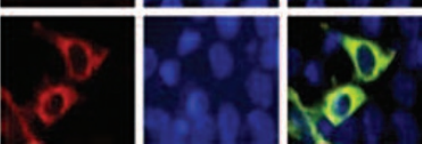
Furthermore, we found that RBP-Jk inhibited the scaffold activity of JIP1 in the JNK signaling pathway through physical association. These findings give us interesting evidence for negative feedback between Notch1 and JIP1 signaling.

Our results showed that Notch1 transcriptional activity was inhibited by JIP1 in intact cells and that the transcriptional activity of endogenous Notch1-IC was higher in JIP1 null cells than in JIP1 wild-type cells, suggesting that JIP1 can regulate Notch1-IC signaling. As the nucleus constitutes the primary site of interaction between Notch1-IC and RBP-Jk, and JIP1 is localized primarily in the cytoplasm, in complexes with components of JNK signaling, it is unlikely that JIP1 directly regulates Notch1-IC transcription activity. In our subsequent experiments, we observed that the subcellular location of RBP-Jk proteins was predominantly in the cytoplasm in the presence of JIP1, which indicated that JIP1 may regulate the subcellular distribution of RBP-Jk. The subcellular distribution of Notch1-IC was not significantly alerted by JIP1. Our data imply that JIP1 may suppress Notch1 transcriptional activity through cytoplasmic retention of RBP-Jk (Figure 7a).

In this study, we observed both in vivo and in vitro binding between RBP-Jk and JIP1 proteins. We also confirmed both in vivo and in vitro binding between Notch $1-\mathrm{IC}$ and JIP1 proteins as reported earlier. ${ }^{17}$ The interaction between JIP1 and either RBP-Jk or Notch1-IC protein was not enhanced by the presence of both proteins together. Furthermore, we also found the formation of the JIP1-Notch1-IC-RBP-Jk trimeric complex in this case. In the trimeric complex, we questioned whether JIP1 could bind RBP-Jk without Notch1-IC, or whether JIP1 could bind Notch1-IC without RBP-Jk. By using Notch1-IC $\triangle$ RAM, which lacks the RBP-Jk-interacting domain, we proved that, while JIP1 can form a trimeric complex with Notch 1-IC and RBP-Jk, it binds them at distinct sites despite this trimeric complex formation. Notch1-IC could be localized into the nucleus in the presence of JIP1, implying that binding between JIP1 and Notch1-IC is not persistently kept. On the basis of these results, we propose that endogenous JIP1 may prevent Notch1 transcriptional activity through the sequestering of RBP-Jk, because of the dissociation of Notch1-IC and RBP-Jk.

JIP1 contains an N-terminal JBD and C-terminal MLK- and MKK7-binding domain. The C-terminal MLK- and MKK7binding domain contains a putative PID and an $\mathrm{SH} 3$ domains. ${ }^{21,23}$ JBD binding, in particular, has pivotal functions in binding with JNK. In our earlier reports, we have shown that the JBD domain of JIP1 is the binding site for Notch1-IC. ${ }^{17}$ Interestingly, our results seem to show that the $\triangle \mathrm{JBD}$ domain of JIP1 including SH3 domain is capable of inhibiting Notch1 transcriptional activity through direct binding, whereas the JBD and PID-KE domains alone neither bind to Notch1, nor inhibit Notch1 transcriptional activity. Accordingly, Notch1-IC and RBP-Jk bind to distinct regions of JIP1. SH3 domains mediate the assembly of specific protein complexes through binding to proline-rich peptides in their respective binding partners. ${ }^{24}$ We found that JIP1 did not alter the RBP-Jk (PPP/AAA) mutant and Notch1-IC-mediated transactivation, and subcellular localization of RBP-Jk (PPP/AAA) mutant. Here, we report that maybe the proline-rich region of RBP-Jk is recognized by the SH3 domain of JIP1. Thus, the SH3 domain is important for the binding of JIP1 to RBP-Jk and the JIP1-induced inhibition of Notch1 signaling.

Notch1 has a critical function in the determination of cell fate, and in many cases this is related to the regulation of apoptosis. Interestingly, according to a study by MacKenzie et al. ${ }^{25}$, the inhibition of JNK activation and cell death by Notch4 activation in endothelial cells is through RBP-Jkdependent and -independent pathways, ${ }^{25}$ whereas a recent report showed that the activation of RBP-Jk-dependent Notch1 signaling on its own is sufficient to prevent druginduced JNK activation and cell death. ${ }^{26}$ Our earlier work shows that Notch1-IC, by binding to JIP1, interferes with the physical interaction between JIP1 and JNK, thereby antagonizing the JIP1-mediated activation of JNK signaling. ${ }^{17}$ Taken together, these earlier reports raise the question of whether RBP-Jk is a critical player in JIP1-mediated activation of JNK signaling and cell death. We observed that RBP-Jk interferes with the physical association of JIP1 with MLK3 and MKK7, but not with JNK (Figure 7b). Given that Notch1-IC and RBP$\mathrm{Jk}$ bind to distinct regions of JIP1, and that both of the proteins have anti-apoptotic functions, it would be worthwhile to examine the possibility of functional cooperation or compensation between Notch1 and RBP-Jk for regulating JIP1mediated JNK activation and cell death. It would also be of considerable interest to determine whether RBP-Jk, such as Notch1-IC, has a protective function in excitotoxic neuronal injury. The results of this study shed light on a signal cross-talk

Figure 5 C-terminal region of JIP1 including the SH3 domain is critical for binding to RBP-Jk. (a) Recombinant GST and GST-RBP-Jk proteins were immobilized onto GSH-agarose. HEK293 cells were transfected for $48 \mathrm{~h}$ with expression vectors encoding Flag-JIP1, Flag-JIP1- $\Delta J \mathrm{JBD}$, Flag-JIP1-JBD, Flag-JIP1-PID, or empty vector. After transfection, the cell lysates were then subjected to GST-pull-down experiments with immobilized GST or GST-RBP-Jk. Proteins bound to GST or GST-RBP-Jk were analyzed through immunoblotting with anti-Flag antibody. Cell lysates were also immunoblotted with anti-Flag antibody. (b) HEK293 cells were transfected for $48 \mathrm{~h}$ with expression vectors encoding empty vector, Flag-JIP1, Flag-JIP1- $\triangle J B D$, Flag-JIP1-JBD, and Flag-JIP1-PID. After transfection, the cell lysates were subjected to immunoprecipitation with anti-RBP-Jk antibody. The immunoprecipitates were then immunoblotted with anti-Flag antibody. Cell lysates were also immunoblotted with anti-RBP-Jk and anti-Flag antibodies. (c) Schematic diagram of the JIP1 polypeptide, and sites of its physical binding with RBP-Jk and Notch1-IC proteins. The regions of JIP1 that interact with RBP-Jk and Notch1-IC are shown. In our earlier report, it was shown that Notch1-IC could bind to the JBD domain of JIP1. ${ }^{24} \mathrm{JBD}$, JNK-binding domain; SH3, src homology domain 3 ; PID, phosphotyrosine interaction domain. (d) NIH3T3 cells were transfected with expression vectors for 4XCSL-Luc, and $\beta$-galactosidase, along with Notch1-IC and JIP1 deletion mutants ( $\triangle J B D$ and PID), as indicated. (e) HEK293 cells were transfected for $48 \mathrm{~h}$ with expression vectors encoding Flag-RBP-Jk, Flag-RBP-Jk (PPP/AAA) mutant (M, mutant), and HA-JIP1. After transfection, the cell lysates were subjected to immunoprecipitation with anti-HA antibody. The immunoprecipitates were then immunoblotted with anti-Flag antibody. Cell lysates were also immunoblotted with anti-Flag and anti-HA antibodies. (f) HEK293 cells were transfected with expression vectors for 4XCSL-Luc, and $\beta$-galactosidase, along with Notch1-IC, RBP-Jk, RBP-Jk (PPP/AAA), and JIP1- $\triangle \mathrm{JBD}$, as indicated. (d, f) After $48 \mathrm{~h}$ of transfection, the cells were lysed, and the luciferase activity was determined. The data were normalized with $\beta$-galactosidase. These results represent the means \pm average deviation of three independent experiments. RLU means relative luciferase units. (g) HEK293 cells were transfected with expression vectors for Flag-RBP-Jk, Flag-RBP-Jk (PPP/AAA), and HA-JIP1, as indicated. The cells were double stained for RBP-Jk (green) and JIP1 (red). (h) HEK293 cells were transfected with expression vectors for HA-RBP-Jk and Flag-JIP1 wild type and Flag-JIP1 $\triangle \mathrm{JBD}$, as indicated. The cells were double stained for RBP-Jk (green) and JIP1 (red). W, wild type; M, mutant 


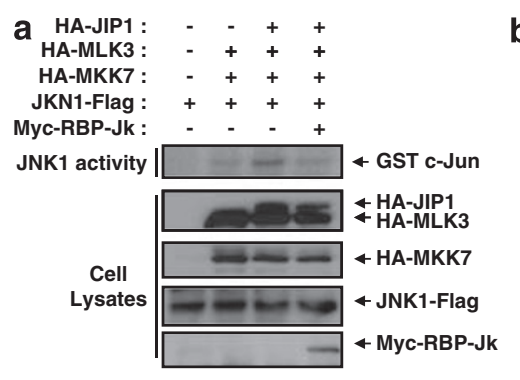

d

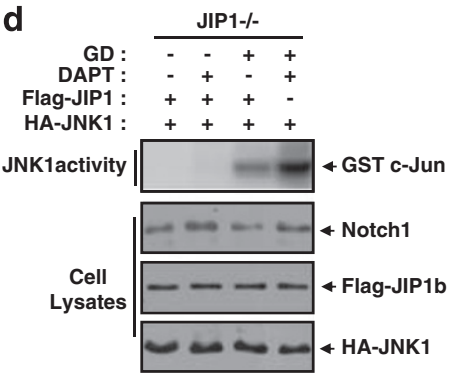

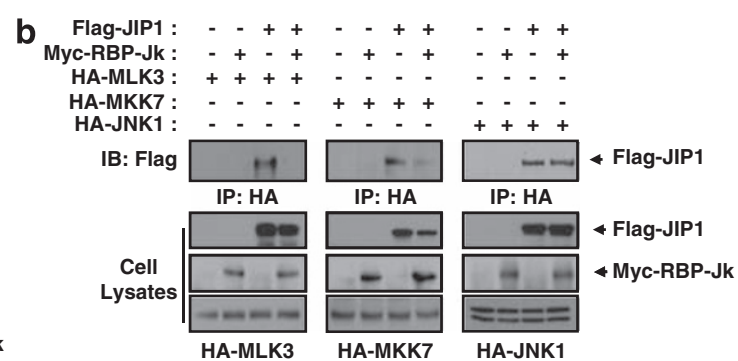

C
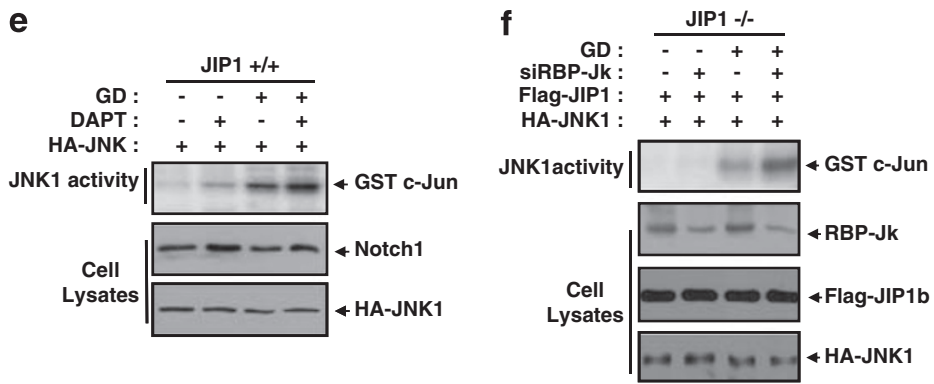

g

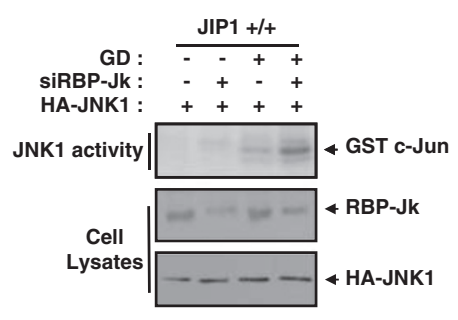

$\mathbf{h}$
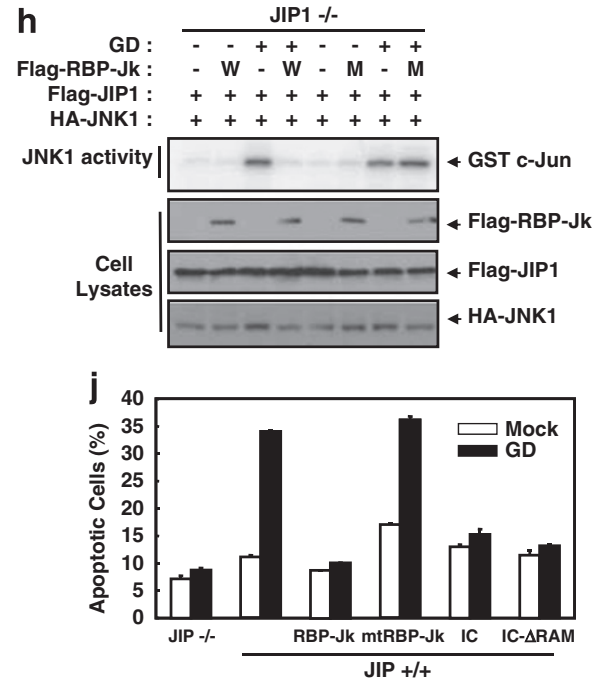

i

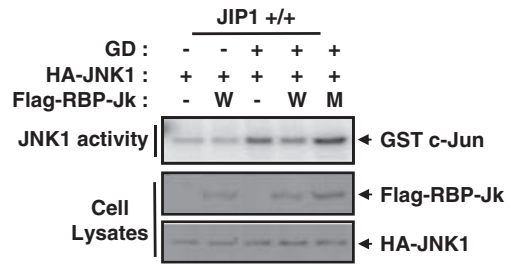

Figure 6 RBP-Jk prevents JIP1-mediated signaling and cell death. (a) HEK293 cells were transfected for $48 \mathrm{~h}$ with expression vectors encoding empty vector, HA-JIP1, HA-MLK3, HA-MKK7, Flag-JNK1, and Myc-RBP-Jk. After transfection, the cells were lysed and immunoprecipitation with anti-Flag antibody and the resulting precipitates were assayed for JNK1 activity. The cell lysates were immunoblotted with anti-HA, anti-Flag, and anti-Myc antibodies. (b) HEK293 cells were transfected for $48 \mathrm{~h}$ with expression vectors encoding Flag-JIP1, Myc-RBP-Jk, HA-MLK3, HA-MKK7, and HA-JNK1, as indicated. After transfection, the cell lysates were subjected to immunoprecipitation with anti-HA antibody. The immunoprecipitates were then immunoblotted with anti-Flag antibody. The cell lysates were also immunoblotted with anti-Flag, anti-Myc, and anti-HA antibodies. (c) HEK293 cells were transfected for $48 \mathrm{~h}$ with expression vectors encoding empty vector, HA-JIP1, Flag-JIP1, and Myc-RBP-Jk. After transfection, the cell lysates were subjected to immunoprecipitation with anti-Flag antibody. The immunoprecipitates were then immunoblotted with anti-HA antibody. Cell lysates were also immunoblotted with anti-Flag, anti-HA, and anti-Myc antibodies. (d) JIP1 $1^{-1}$ MEF cells were transfected for $48 \mathrm{~h}$ with expression vectors encoding Flag-JIP1 and HA-JNK1. The transfected cells were treated with $1 \mathrm{mM}$ of DAPT for $4 \mathrm{~h}$. (e) JIP1 ${ }^{+/+}$MEF cells were transfected for $48 \mathrm{~h}$ with expression vectors encoding HA-JNK1. The transfected cells were treated with $1 \mathrm{mM}$ of DAPT for $4 \mathrm{~h}$. (f) JIP1 ${ }^{-l-}$ MEF cells were transfected for $48 \mathrm{~h}$ with expression vectors encoding siRBP-Jk, Flag-JIP1, and HA-JNK1. (g) JIP1 ${ }^{+1+}$ MEF cells were transfected for $48 \mathrm{~h}$ with expression vectors encoding siRBP-Jk and HA-JNK1. (h) JIP1 ${ }^{-/-}$MEF cells were transfected for $48 \mathrm{~h}$ with expression vectors encoding Flag-RBP-Jk, Flag-RBP-JK (PPP/AAA), Flag-JIP1, and HA-JNK1. (i) JIP1 ${ }^{+/+}$MEF cells were transfected for $48 \mathrm{~h}$ with expression vectors encoding HA-JNK1, Flag-RBP-JK, and FlagRBP-Jk (PPP/AAA). (d-i) The transfected cells were incubated first for $4 \mathrm{~h}$ in DMEM (serum-free) or glucose-free DMEM (serum-free) and then for an additional $12 \mathrm{~h}$ in complete medium. Cell lysates were then subjected to immunoprecipitation with anti-HA antibody and the resulting precipitates were assayed for JNK1 activity. The cell lysates were immunoblotted with anti-Myc, anti-Flag, anti-HA, anti-Notch1, or anti-RBP-Jk antibodies. (j) JIP1 ${ }^{-1-}$ and JIP1 ${ }^{+1+}$ MEF cells were transfected for $48 \mathrm{~h}$ with eGFP alone or together with an expression vector encoding RBP-Jk (WT), RBP-Jk (MT), Notch1-IC, and Notch1-IC- $\triangle$ RAM. The transfected cells were incubated first for $4 \mathrm{~h}$ in DMEM (serum-free) or glucose-free DMEM (serum-free) and then for an additional $24 \mathrm{~h}$ in complete medium. The cells were then stained with DAPI, and TUNEL and GFP-positive cells were scored for apoptotic nuclei with a fluorescence microscope. Data are means \pm average deviation of values from three independent experiments. GD, glucose deprivation; mtRBP, RBP-Jk (PPP/AAA) 
a
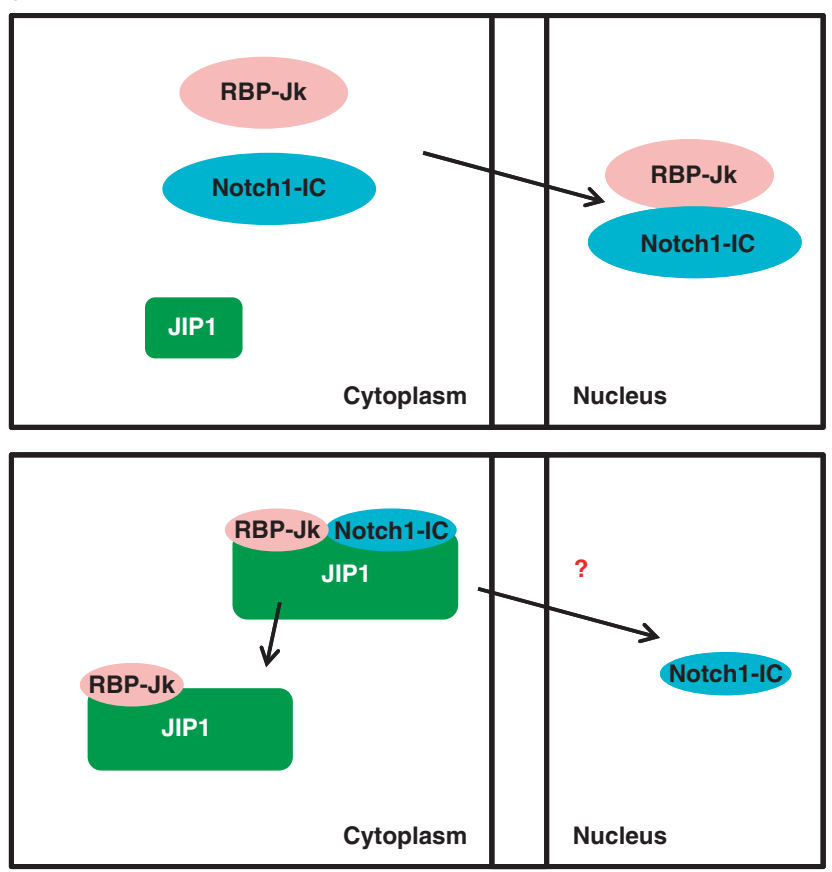

b

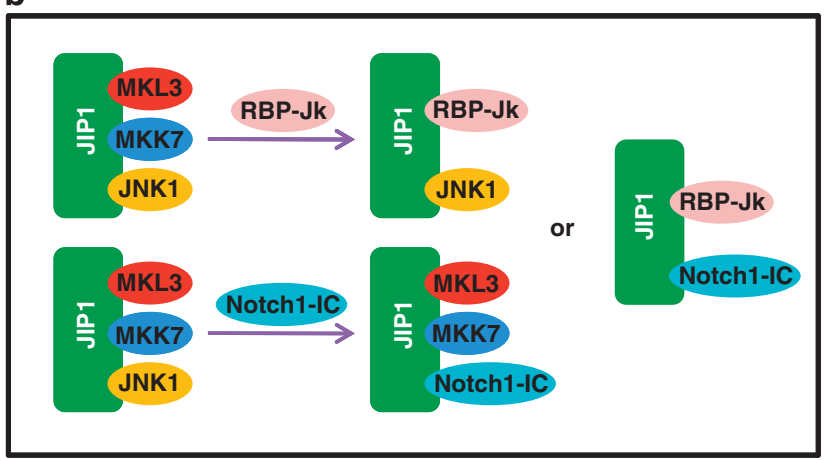

Figure 7 Model for the signal cross-talk between Notch-RBP-Jk and JIP1-JNK1 signaling pathway. (a) JIP1 contains an N-terminal JNK-binding domain (JBD) and C-terminal MLK- and MKK7-binding domain. In our earlier reports, we have shown that the JBD domain of JIP1 is the binding site for Notch1-IC and thereby prevent JNK1 binding with JBD domain. ${ }^{17}$ SH3 domain of JIP1 is recognized by the proline-rich region of RBP-Jk. RBP-Jk interferes with the physical association of JIP1 with MLK3 and MKK7, but not with JNK1. Given that Notch1-IC and RBP-Jk bind to distinct regions of JIP1, and that both of the proteins have antiapoptotic functions. (b) At the low level of JIP1 expression, Notch1-IC interacts with RBP-Jk and with coactivator complexes to promote target gene expression. At the high level of JIP1 expression, the RBP-Jk proteins are accumulated in the cytoplasm after it forms a trimeric complex with JIP1 and Notch1. After binding with JIP1, however, Notch-IC translocates to the nucleus with unknown mechanism thereby forms a decreased Notch1-RBP-Jk complex by JIP1 in the nucleus

mechanism of RBP-Jk and JIP1, or they may point to the existence of Notch1-independent cross-talk in the regulation of cell-fate decisions, differentiation, proliferation, and cell death and survival.

\section{Materials and Methods}

Cell culture and transfection. HEK293 cells, NIH3T3 cells, PC12 cells, SH-SY5Y cells, and MEFs from JIP1 $1^{+1+}$ or $\mathrm{JIP} 1^{-1-}$ mice were maintained at $37^{\circ} \mathrm{C}$ in Dulbecco's Modified Eagle's Medium (DMEM) supplemented with $10 \%$ fetal bovine serum and $1 \%$ penicillin/streptomycin, in a humidified incubator with an atmosphere containing $5 \% \mathrm{CO}_{2}$. The $\mathrm{JPP} 1^{+/+}$and $\mathrm{JIP} 1^{-1-} \mathrm{MEF}$ cells were used at passages from 6 to 10 , and they were in the same passage at the experiments. The cultured cells were transiently transfected by the calcium phosphate method or Lipofectamine (Invitrogen, Camarillo, CA, USA). ${ }^{17,18,27}$

Cloning and preparation of recombinant proteins. Mouse Notch1-IC and RBP-Jk CDNAs and deletion mutants were constructed through standard PCR, and inserted into the bacterial expression vector, pGEX4T-3 (Amersham Pharmacia, Piscataway, NJ, USA) as described earlier. ${ }^{28,29}$ The expression of the recombinant GST-Notch1-IC and GST-RBP-Jk proteins within the transformed bacteria was induced using $1 \mathrm{mM}$ isopropyl- $\beta$-D-thiogalactopyranoside (Sigma, St Louis, MO, USA). The GST-Notch1-IC and GST-RBP-Jk proteins were purified with GSH-agarose (Sigma), in accordance with the manufacturer's instructions.

Site-directed mutagenesis. Site-directed mutagenesis of RBP-Jk cDNA was performed with a QuikChange kit (Stratagene, La Jolla, CA, USA), and the mutagenic primers were P95/96/97A (5'-CGATTTTTTTGCgCT gCTgCTTGTGTGTATC- ${ }^{\prime}$ ) (mismatches with the RBP-Jk cDNA template are indicated by lowercase letters). The mutations were verified by automatic DNA sequencing.

Luciferase reporter assay. The HEK 293 cells, $\mathrm{NIH} 3 \mathrm{~T} 3$ cells, and JIP1 ${ }^{+/+}$ and $\mathrm{JIP} 1^{-1-}$ MEF cells were cotransfected with 4XCSL-Luc and $\beta$-galactosidase together with the indicated vector constructs. The cells were lysed in chemiluminescent lysis buffer [18.3\% of $1 \mathrm{M} \mathrm{K}_{2} \mathrm{HPO}_{4}, 1.7 \%$ of $1 \mathrm{M} \mathrm{KH}_{2} \mathrm{PO}_{4}, 1 \mathrm{mM}$ phenylmethyl sulfonyl fluoride (PMSF), and $1 \mathrm{mM}$ dithiothreitol (DTT)] and assayed for luciferase activity with a luciferase assay kit (Promega, Madison, WI, USA). The activity of the luciferase reporter protein in the transfected cells was normalized in reference to the $\beta$-galactosidase activity in the same cells. ${ }^{28}$

Western blot analysis. After $48 \mathrm{~h}$ of transfection, the cultured HEK293 cells were harvested and lysed in RIPA buffer ( $50 \mathrm{mM}$ Tris- $\mathrm{HCl}(\mathrm{pH} 7.5), 150 \mathrm{mM} \mathrm{NaCl}$, $1 \%$ nonidet P-40, $0.5 \%$ sodium deoxycholate, $0.1 \%$ SDS, $1 \mathrm{mM} \mathrm{PMSF}, 1 \mathrm{mM}$ DTT, and $2 \mu \mathrm{g} / \mathrm{ml}$ leupeptin and aprotinin) for $30 \mathrm{~min}$. The cell lysates were subjected to $20 \mathrm{~min}$ of centrifugation at $12000 \times \mathrm{g}$ at $4^{\circ} \mathrm{C}$. The resultant soluble fraction was boiled in Laemmli buffer and subjected to SDS-PAGE. After gel electrophoresis, the separated proteins were transferred through electroblotting onto polyvinylidene difluoride membranes (Millipore, Temecula, CA, USA). The membranes were then blocked with Tris-buffered saline solution (pH 7.4) containing $0.1 \%$ Tween 20 and $5 \%$ nonfat milk. The blotted proteins were then probed with anti-Myc antibody (9E10), anti-HA (12CA5) antibody, anti-Flag M2 antibody (Sigma Chemical Co., St Louis, MO, USA), anti-RBP-Jk (Santa Cruz, Santa Cruz, CA, USA), anti-Notch1 (Cell signaling, Danvers, MA, USA; Santa Cruz), anti-JIP1 (Santa Cruz), or anti- $\beta$ actin followed by incubation with anti-mouse horseradish peroxidase-conjugated secondary antibodies (Amersham Biosciences). The blots were developed using an enhanced chemiluminescence system (Pierce, Rockford, IL, USA).

Immunocomplex kinase assay. To analyze the kinase activity, confluent cells were harvested and lysed in RIPA lysis buffer. The cell lysates were then subjected to $10 \mathrm{~min}$ of centrifugation at $12000 \times \mathrm{g}$ at $4^{\circ} \mathrm{C}$. The soluble fractions were incubated for $1 \mathrm{~h}$ with appropriate antibodies against the indicated protein kinases at $4^{\circ} \mathrm{C}$. The immunocomplexes were then coupled to protein $\mathrm{G}$-agarose during an additional $1 \mathrm{~h}$ of incubation at $4^{\circ} \mathrm{C}$, after which they were pelleted through centrifugation. The immunopellets were rinsed three times with lysis buffer and then twice with $20 \mathrm{mM}$ Hepes, at a $\mathrm{pH}$ of 7.4. Immunocomplex kinase assays were conducted through the incubation of the immunopellets for $30 \mathrm{~min}$ at $30^{\circ} \mathrm{C}$ with $2 \mu \mathrm{g}$ of substrate proteins in $20 \mu \mathrm{l}$ of reaction buffer containing $0.2 \mathrm{mM}$ sodium orthovanadate, $10 \mathrm{mM} \mathrm{MgCl}_{2}, 2 \mu \mathrm{Ci}\left[\gamma^{32} \mathrm{P}\right] \mathrm{ATP}$, and $20 \mathrm{mM}$ Hepes (pH 7.4). The phosphorylated substrates were then visualized through SDS-PAGE, and quantified using a Fuji BAS 2500 phospholmager. ${ }^{29}$

Immunofluorescence staining. The assays were conducted as described earlier, with cells plated at $1 \times 10^{5}$ cells per well onto cover slips (Fisher). A total of $0.5 \mu \mathrm{g}$ of appropriate DNA per well was then transfected with Geneporter2 (Genetherapysystems). The transfected cells were fixed with $4 \%$ paraformaldehyde in phosphate-buffered saline (PBS), and then permeabilized with $0.1 \%$ Triton X-100 in PBS. Mouse anti-Flag M2 antibody (Sigma), anti-HA antibody (Sigma), and anti-Myc 9E10 antibody (Novus Biologicals, Littleton, CO, USA) were used as the primary antibodies at a dilution of $1: 100$. Either Rhodamine Red or 
fluorescein-conjugated anti-mouse secondary antibody (1:100) was added, followed by staining with DAPI. The stained cells were evaluated for localization through confocal microscopy (Leica TCS SPE). ${ }^{19}$

In vitro binding assay. The recombinant GST-Notch1-IC and GST-RBP-Jk proteins were expressed in Escherichia coli strain BL21, using the pGEX system as indicated. The GST fusion proteins were then purified using glutathione-agarose beads (Sigma), in accordance with the manufacturer's instructions. Equal amounts of the GST fusion proteins were incubated with transfected cell lysates for $3 \mathrm{~h}$ at $4{ }^{\circ} \mathrm{C}$, with rotation. After incubation, the beads were washed three times with ice-cold PBS, and boiled with $20 \mu \mathrm{l}$ of Laemmli sample buffer. The precipitates were separated through SDS-PAGE, and the pull-down proteins were detected through immunoblotting with specific antibodies. ${ }^{30}$

Coimmunoprecipitation assays. The cells were lysed in $1 \mathrm{ml}$ of RIPA buffer for $30 \mathrm{~min}$ at $4^{\circ} \mathrm{C}$. After centrifugation at $12000 \times \mathrm{g}$ for $20 \mathrm{~min}$, the supernatants were subjected to immunoprecipitation with appropriate antibodies coupled to protein A-agarose beads. Preparation of cytoplasmic fraction was conducted as described earlier. ${ }^{17-19,31}$ The resulting immunoprecipitates were washed three times with phosphate-buffered solution (PBS, pH 7.4). Laemmli sample buffer was then added to the immunoprecipitated pellets; the pellets were heated at $95^{\circ} \mathrm{C}$ for $5 \mathrm{~min}$ and then analyzed by SDS-PAGE. The western blot was performed with the indicated antibodies. ${ }^{28}$

Apoptotic cell death. Cultured cells were cotransfected with appropriate plasmid vectors plus pEGFP using Lipofectamine (Gibco BRL, Carlsbad, CA, USA). After $50 \mathrm{~h}$ of transfection, the cells were fixed with $0.25 \%$ glutaraldehyde and stained with DAPI. Apoptotic cell death was also analyzed by the terminal deoxynucleotidyl transferase-mediated dUTP nick end-labeling (TUNEL) assay. The TUNEL staining was carried out using the in situ cell death detection kit (Roche Molecular Biochemicals, Chicago, IL, USA) according to the manufacturer's protocol. Briefly, cells in culture were fixed with $4 \%$ paraformaldehyde, permeabilized with $0.1 \%$ Triton X-100/0.1\% sodium citrate solution, and then incubated for $60 \mathrm{~min}$ at $37^{\circ} \mathrm{C}$ with TUNEL reaction mixture containing terminal deoxynucleotidyl transferase and fluorescein-labeled dUTP. The TUNEL-positive cells were detected by fluorescence microscopy. ${ }^{17-19}$

\section{Conflict of interest}

The authors declare no conflict of interest.

Acknowledgements. We thank Roger J Davis (University of Massachusetts Medical School, Worcester), JR Woodgett (Ontario Cancer Institute, Toronto), Raphael Kopan (Washington University, St Louis), Alan Israël (Institut Pasteur, Paris), Tasuku Honjo (Kyoto University, Kyoto) for providing JNK1, SAPKb, Notch1, and RBP-Jk cDNA clones. This study was supported by a grant of the Korea Healthcare technology R\&D Project, Ministry of Health \& Welfare, Republic of Korea (A08-4065).

1. Artavanis-Tsakonas $S$, Rand MD, Lake RJ. Notch signaling: cell fate control and signal integration in development. Science 1999; 284: 770-776.

2. Weinmaster G. The ins and outs of notch signaling. Mol Cell Neurosci 1997; 9: 91-102.

3. Kopan R, llagan MX. The canonical Notch signaling pathway: unfolding the activation mechanism. Cell 2009; 137: 216-233.

4. Kao HY, Ordentlich P, Koyano-Nakagawa N, Tang Z, Downes M, Kintner CR et al. A histone deacetylase corepressor complex regulates the Notch signal transduction pathway. Genes Dev 1998; 12: 2269-2277.

5. Whitmarsh AJ. The JIP family of MAPK scaffold proteins. Biochem Soc Trans 2006; 34 (Part 5): 828-832.

6. Davis RJ. Signal transduction by the JNK group of MAP kinases. Cell 2000; 103: 239-252.
7. Matsuda S, Yasukawa T, Homma Y, Ito Y, Niikura T, Hiraki T et al. c-Jun N-terminal kinase (JNK)-interacting protein-1b/islet-brain-1 scaffolds Alzheimer's amyloid precursor protein with JNK. J Neurosci 2001; 21: 6597-6607.

8. Meyer D, Liu A, Margolis B. Interaction of c-Jun amino-terminal kinase interacting protein-1 with p190 rhoGEF and its localization in differentiated neurons. J Biol Chem 1999; 274: 35113-35118.

9. Verhey KJ, Meyer D, Deehan R, Blenis J, Schnapp BJ, Rapoport TA et al. Cargo of kinesin identified as JIP scaffolding proteins and associated signaling molecules. J Cell Biol 2001; 152: 959-970.

10. Stockinger W, Brandes C, Fasching D, Hermann M, Gotthardt M, Herz J et al. The reelin receptor ApoER2 recruits JNK-interacting proteins-1 and -2. J Biol Chem 2000; 275: 25625-25632.

11. Oh SM, Zhu F, Cho YY, Lee KW, Kang BS, Kim HG et al. T-lymphokine-activated killer celloriginated protein kinase functions as a positive regulator of c-Jun-NH2-kinase 1 signaling and H-Ras-induced cell transformation. Cancer Res 2007; 67: 5186-5194.

12. Santos $C R$, Blanco $S$, Sevilla A, Lazo PA. Vaccinia virus B1R kinase interacts with JIP1 and modulates c-Jun-dependent signaling. J Virol 2006; 80: 7667-7675.

13. Blanco S, Sanz-Garcia M, Santos CR, Lazo PA. Modulation of interleukin-1 transcriptional response by the interaction between VRK2 and the JIP1 scaffold protein. PLoS One 2008; 3: e1660.

14. Song JJ, Lee YJ. Dissociation of Akt1 from its negative regulator JIP1 is mediated through the ASK1-MEK-JNK signal transduction pathway during metabolic oxidative stress: a negative feedback loop. J Cell Biol 2005; 170: 61-72.

15. Nihalani D, Wong H, Verma R, Holzman LB. Src family kinases directly regulate JIP1 module dynamics and activation. Mol Cell Biol 2007; 27: 2431-2441.

16. Blanco S, Santos C, Lazo PA. Vaccinia-related kinase 2 modulates the stress response to hypoxia mediated by TAK1. Mol Cell Biol 2007; 27: 7273-7283.

17. Kim JW, Kim MJ, Kim KJ, Yun HJ, Chae JS, Hwang SG et al. Notch interferes with the scaffold function of JNK-interacting protein 1 to inhibit the JNK signaling pathway. Proc Natl Acad Sci USA 2005; 102: 14308-14313.

18. Kim SY, Kim MY, Mo JS, Park HS. Notch1 intracellular domain suppresses APP intracellular domain-Tip60-Fe65 complex mediated signaling through physical interaction. Biochim Biophys Acta 2007; 1773: 736-746.

19. Mo JS, Kim MY, Ann EJ, Hong JA, Park HS. DJ-1 modulates UV-induced oxidative stress signaling through the suppression of MEKK1 and cell death. Cell Death Differ 2008; 15 : 1030-1041.

20. Kovall RA, Hendrickson WA. Crystal structure of the nuclear effector of Notch signaling, CSL, bound to DNA. EMBO J 2004; 23: 3441-3451.

21. Dickens M, Rogers JS, Cavanagh J, Raitano A, Xia Z, Halpern JR et al. A cytoplasmic inhibitor of the JNK signal transduction pathway. Science 1997; 277: 693-696.

22. Whitmarsh AJ, Kuan CY, Kennedy NJ, Kelkar N, Haydar TF, Mordes JP et al. Requirement of the JIP1 scaffold protein for stress-induced JNK activation. Genes Dev 2001; 15 : 2421-2432.

23. Whitmarsh AJ, Davis RJ. Structural organization of MAP-kinase signaling modules by scaffold proteins in yeast and mammals. Trends Biochem Sci 1998; 23: 481-485.

24. Feng S, Kasahara C, Rickles RJ, Schreiber SL. Specific interactions outside the prolinerich core of two classes of Src homology 3 ligands. Proc Natl Acad Sci USA 1995; 92: 12408-12415.

25. MacKenzie F, Duriez P, Wong F, Noseda M, Karsan A. Notch4 inhibits endothelial apoptosis via RBP-Jkappa-dependent and -independent pathways. J Biol Chem 2004; 279: 11657-11663.

26. Stylianou S, Clarke RB, Brennan K. Aberrant activation of notch signaling in human breast cancer. Cancer Res 2006; 66: 1517-1525.

27. Im JY, Lee KW, Kim MH, Lee SH, Ha HY, Cho IH et al. Repression of phospho-JNK and infarct volume in ischemic brain of JIP1-deficient mice. J Neurosci Res 2003; 74: 326-332.

28. Kim MY, Ann EJ, Kim JY, Mo JS, Park JH, Kim SY et al. Tip60 histone acetyltransferase acts as a negative regulator of Notch1 signaling by means of acetylation. Mol Cell Biol 2007: 27: 6506-6519.

29. Mo JS, Kim MY, Han SO, Kim IS, Ann EJ, Lee KS et al. Integrin-linked kinase controls Notch1 signaling by down-regulation of protein stability through Fbw7 ubiquitin ligase. Mol Cell Biol 2007; 27: 5565-5574.

30. Kim MY, Park JH, Mo JS, Ann EJ, Han SO, Baek SH et al. Downregulation by lipopolysaccharide of Notch signaling, via nitric oxide. J Cell Sci 2008; 121 (Part 9): 1466-1476.

31. Park HS, Lee JS, Huh SH, Seo JS, Choi EJ. Hsp72 functions as a natural inhibitory protein of c-Jun N-terminal kinase. EMBO J 2001; 20: 446-456. 